\title{
SPECTROSCOPIC DIAGNOSIS OF SN1987A AND LESSER LIGHTS
}

\section{J. Craig Wheeler, Robert P. Harkness \\ Department of Astronomy \\ University of Texas at Austin}

Zalman Barkat

Department of Physics

Hebrew University of Jerusalem

\begin{abstract}
SN 1987A gives a unique chance to study both the precursor star and the subsequent dynamical evolution of the explosion. Comparison of the light curves shows that elther $H_{0} \sim 100 \mathrm{~km} / \mathrm{s} / \mathrm{Mpc}$, or SN $1987 \mathrm{~A}$ ejected significantly less ${ }^{56} \mathrm{NI}$ than ordinary Type II supernovae. Investigation of the stellar structure pertinent to SK -69202 reveals multiple solutions. For given luminosity, effective temperature, core mass and core radius, there are two families of envelope mass, one with large envelope mass and one with small envelope mass. The small envelope mass solutions can be ruled out by considerations of kinematics and the light curve. Envelopes of moderate mass may avoid each of these problems, but must be helium rich to be structurally self-consistent.

The spectrum in both the optical and the ultraviolet at about two days is fairly well represented by a hydrogen envelope with a power law density profile $\left(\rho \propto r^{-11}\right)$ of one-quarter solar metallicity In LTE. Theoretical spectra at this early epoch tend to favor luminosities on the high side of observational estimates in order to lonize $\mathrm{Ca}$ II and prevent excessively strong lines at $H$ and $K$ and the Infrared triplet, with some ramifications for distance estimates.

The spectra of SN1987A present an Interesting contrast to other SN II events. A McDonald Observatory spectrum of SN 1985H in NGC 3359 of uncertain epoch shows a very close resemblance to that of SN 1987A at about two month's age, including the strong line at $607 \mathrm{~nm}$ attributed by Williams to Barlum. SN $1985 \mathrm{H}$ may have been of the same class of event as SN 1987A.
\end{abstract}




\section{INTRODUCTION}

SN 1987A has confirmed some of the most basic predictions of supernova theory, but has also presented unique aspects which project supernova research in exciting new directions guided by an impressive wealth of detailed observations. This situation provides a marvelous laboratory to test a wide variety of theories, assumptions, tech-

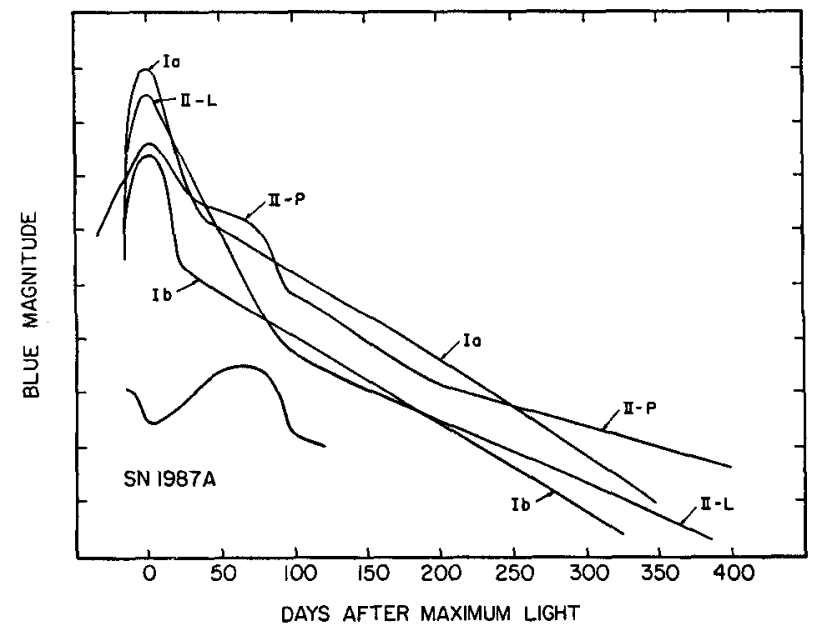

Figure 1. Schematic B magnitude light curves are presented for supernovae of Type la, Ib, II linear, and II plateau in contrast with that of SN 1987A. The absolute magnitude of SN 1987A is relatively well known. The displacement with respect to the other types assumes a distance scale corresponding to $\mathrm{H}_{0}=50$ $\mathrm{km} / \mathrm{s} / \mathrm{Mpc}$.

niques, and relations. In this paper we discuss some aspects of the structure of the progenitor star, including multiple solutions of the stellar structure equations and the controversy over the mass of the hydrogen envelope, the potential of supernova atmosphere calculations to give a new dimension to the analysis of Type II supernovae, and a comparison of SN 1987A with other Type II supernovae.

To put SN 1987A in perspective, the evolving B magnitude light curve is presented in Figure 1 with schematic light curves of the other classes of supernovae adapted from Doggett and Branch(1986), but normalized to show the differences in relative peak magnitudes. Type la are the brightest with some Type II linear events approaching similar luminosity, but also showing a dispersion downward to the magnitudes characteristic of Type II plateau and Type lb events. The long term tails in this figure are not to be taken too literally, since they are statistical, and may contain bias from background galaxy light. In Figure 1, SN 1987A has been dereddened by $0.6 \mathrm{~m}$. This plot suggests that there is little relation between SN 1987A and the other classes of supernovae. This conclusion is in striking contrast to that reached by Peter Wood (private communication) and others, that the declining part of the light curve of $\mathrm{SN}$ 1987A matches rather precisely onto the plateau and tail phase of Type II plateau 
supernovae. There are some minor differences in assumptions and one major one that lead to these different conclusions. One is that there is some uncertainty in the zero point of time, and this can be adjusted to make the fit look as good as possible. In addition, Wood plotted $V$ magnitude light curves. SN 1987A shows a strong UV deficiency, discussed in Section 3, which may account for some of the disparity in magnitude shown in Figure 1. The major difference is that while the "standard" light curves in Figure 1 are plotted only relative to one another, an implicit choice of Hubble constant of about $50 \mathrm{~km} / \mathrm{s} / \mathrm{Mpc}$ was made for them in plotting the relative amplitude of SN 1987A. Wood, in contrast, implicitly assumed a Hubble constant of $100 \mathrm{~km} / \mathrm{s} / \mathrm{Mpc}$. This exercise thus leads us to one of two important conclusions. Either the congruity of light curves noted by Wood is accepted and the Hubble constant is about $100 \mathrm{~km} / \mathrm{s} / \mathrm{Mpc}$, or "normal" Type II-P events are intrinsically brighter than SN 1987A on the tail, suggesting that they eject about 4 times as much $56 \mathrm{Ni}$ as $\mathrm{SN} 1987 \mathrm{~A}$. Both of these possibilities deserve careful consideration.

\section{PROGENITOR STRUCTURE}

The advent of SN 1987A and the very probable identification of its progenitor, SK 69202 , give an unprecedented opportunity to explore the structure of a supernova progenitor. The spatial agreement of SK -69 202 and the supernova, and its disappearance in the UV (Walborn, et al. 1987, Kirshner et al. 1987) strongly argue that this blue supergiant was the progenitor star. Furthermore, models of the light curve constrain the luminosity and radius of the progenitor to be very similar to those observed for SK -69 202 (Arnett 1987a, b, Woosley et al. 1987, Woosley, Pinto and Ensman 1987, Shigeyama, et al. 1987, Grassberg et al. 1987, Hillebrandt et al. 1987). There is still considerable uncertainty about the mass of the hydrogen envelope, with some authors arguing for about $10 \mathrm{M}_{\odot}$ (e.g. Arnett 1987b, Woosley, Pinto and Ensman 1987), and others advocating a rather small envelope, of order $0.1 \mathrm{M}_{\odot}$ (Wood and Faulkner 1987, Maeder 1987). Within the range of assigned luminosities for SK -69202, depending on the distance to the LMC and extinction, plausible progenitors have original main sequence mass in the range roughly 15 to $20 \mathrm{M}_{\odot}$ with helium cores in the range 4 to 6 $M_{\odot}$. We emphasize, however, that only the luminosity and radius (or effective temperature) are directly observationally constrained. The mass of the star and that of the inner core are not known without recourse to solution of the equations of stellar structure and evolution.

In order to understand the physical structure of the progenitor of SN 1987A, models have been constructed which match the radius, effective temperature, and luminosity of SK -69202 by integrating the equations of stellar structure inward from the surface. The composition is assumed to be $X=0.70, Y=0.295$, and $Z=0.005$ for most of the models, although the sensitivity to changes in $X, Y$, and $Z$ has been explored. Details will be presented in Barkat and Wheeler (1988). 
In the current models constructed by inward integration, the composition is specified so that the place where the composition changes at the helium core, either suddenly or gradually, is a free parameter. The specification of the helium core mass implies that the temperature at larger Lagrangian mass may not exceed the threshold temperature for hydrogen burning $\left(\sim 4 \times 10^{7} \mathrm{~K}\right)$. This criterion will be used as a measure of selfconsistency of the models. The bolometric luminosity of SK -69 202 depends on the adopted distance and reddening, with estimates ranging from $L=60,000$ to $100,000 L_{0}$ (Arnett 1987a, Woosley et al. 1987). Models with $\mathrm{M}_{\mathrm{He}}=4$ and $6 \mathrm{M}_{\odot}$ have been explored in some detail to span a reasonable range in allowed parameters. For the models constructed here we take $L=55,000 L_{\odot}$ with a helium core mass of $4 \mathrm{M}_{\odot}$ and $L=$ $110,000 L_{\odot}$ with $\mathrm{MHe}_{\mathrm{He}}=6 \mathrm{M}_{\odot}$.

Evolutionary calculations tend to show that once a core forms, its structure and subsequent evolution are rather independent of the outer envelope. This provides the rationale for studying evolving helium cores (Arnett 1977) without the complication of the envelope. The success of such undertakings gives rise to the intuitive notion that one may construct models of the progenitor of SN 1987A by adopting a core from calculations of evolution at constant mass, and then omitting arbitrary amounts of envelope mass to seek structures that reproduce observations. Woosley, Pinto, and Ensman (1987) have used this technique effectively to explore a large range in parameter space in order to constrain the structures that will reproduce the light curve of SN 1987A. This procedure tacitly assumes that both the core mass and the core radius may be held constant while the luminosity and envelope mass are varied.

Another way of parameterizing the problem of the progenitor structure of SN 1987A is to specify the luminosity, the total mass, and the core mass, and then to examine the behavior of the core radius, $\mathrm{R}_{\mathrm{He}}$, as a function of $\mathrm{T}_{\mathrm{eff}}$ (or $\mathrm{R}$ ). Figure 2 shows the result for models with the standard envelope composition, $M=15 \mathrm{M}_{\odot}$ and $\mathrm{M}_{\mathrm{He}}=4 \mathrm{M}_{\odot}$. For fixed $L, M, M_{H e}$, and $R_{H e}$, if there is a solution with large $T_{\text {eff }}$ then there are, in fact, three solutions to the stellar structure equations, with different values of $T_{\text {eff }}$ and $R$. A similar pattern exists for models with cores of $6 M_{\odot}$. The result is not particularly sensitive to the specific parameters assumed.

The reason for the behavior shown in Figure 2 is illustrated in Figure 3 which gives the plot of the radius, $r$, as a function of the mass, $M_{r}$, for the series of models with $L=$ $55,000 L_{\odot}, M=15 M_{\odot}$, and $M_{H e}=4 M_{\odot}$ for a range in effective temperatures. Note that for large $T_{\text {eff }}$ the gradient in $r$ is rather flat through most of the extent of the mass down to the chosen core mass. For lower $T_{\text {eff }}$ an inflection point is reached before the core is encountered. This causes the curves for lower $T_{\text {eff }}$ to cross those corresponding to higher values, so that two choices of $T_{\text {eff }}$ can give the same value of $R_{\mathrm{He}}$. The reason for this is that the gradient in $r$ with respect to $M_{r}$ gets larger as $T_{\text {eff }}$ decreases and $r^{2} \rho$ decreases. As $T_{\text {eff }}$ decreases even further, the envelope develops convection which flattens out the gradient in $r$, yielding a larger core radius again, and providing the third solution in $T_{\text {eff }}$ for a given value of $R_{\mathrm{He}}$. 


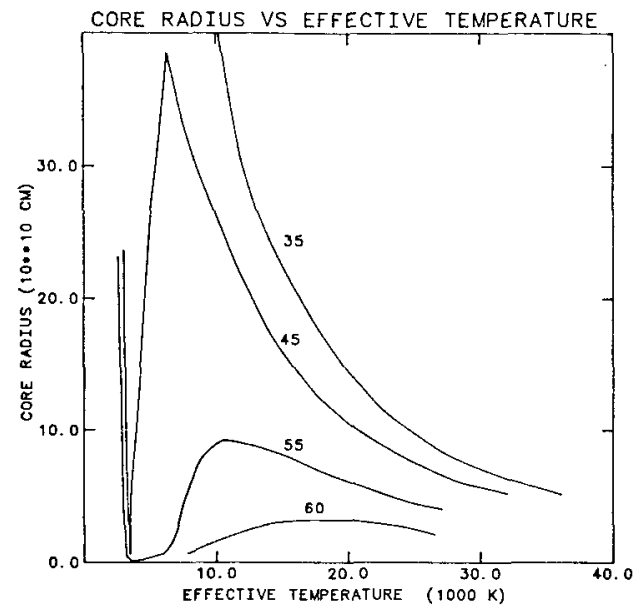

Figure 2. The radius of the core in units of $10^{10} \mathrm{~cm}$ is given as a function of effective temperature for a total mass of $15 \mathrm{M} \odot$ and a helium core mass of $4 \mathrm{M}_{\odot}$. The curves are labeled with the luminosity in units of 1000 Lø. Note that for fixed total mass, core mass, core radius, and luminosity, there are three solutions with different effective temperature. These three solutions have different density structures.

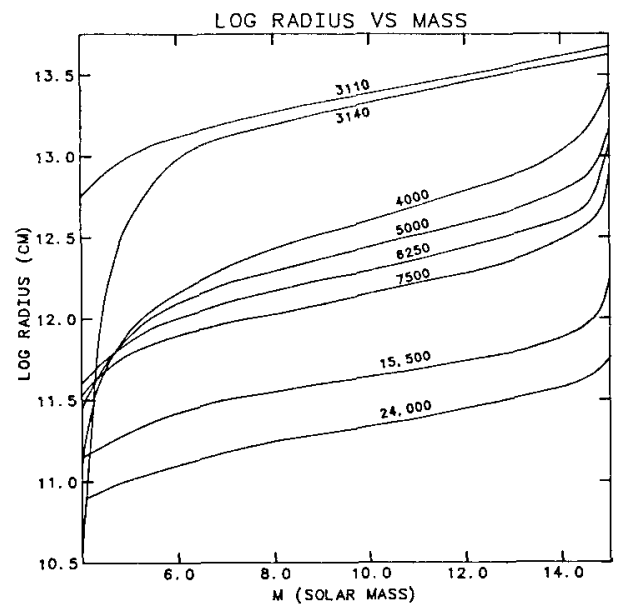

Figure 3. The radius is given as a function of Lagrangian mass for a series of models with total mass $15 \mathrm{M}_{\odot}$, and helium core mass $4 \mathrm{Mo}$, for a range of values of the effective temperature. Note that as the effective temperature declines the temperature gradient steepens giving two solutions with the same core radius, eg, the curves for 15,500 and $4000 \mathrm{~K}$. As the effective temperature continues to decline, the envelope becomes fully convective, and the temperature gradient becomes more shallow, providing the third solution. A model with effective temperature between 3110 and $3140 \mathrm{~K}$ would also have the same core radius as the 15,500 and $4000 \mathrm{~K}$ models. 
The envelope structure for the three solutions at a single core radius are different. In general, the core solutions are similar for the two coolest solutions, and rather different for the bluest solution. For example, for models with $L=55,000 L_{\odot}$ and $R_{H e} \sim 8 \times 10^{10}$ $\mathrm{cm}$ the two cooler models in Figure $2\left(T_{\text {eff }}=3,046 \mathrm{~K}, \mathrm{R} \sim 5 \times 10^{13} \mathrm{~cm}\right.$, and $T_{\text {eff }}=9,500 \mathrm{~K}$, $R \sim 5 \times 10^{12} \mathrm{~cm}$ ) have very similar cores, with the coolest model being on the Hyashi track. The hottest model $\left(T_{\text {eff }}=15,500 \mathrm{~K}, \mathrm{R} \sim 2 \times 10^{12} \mathrm{~cm}\right.$ ) has a central core more nearly equal to the other two models than for a corresponding series with smaller core radius, but the outer core is hotter and denser, as is the envelope, and this may significantly alter the propagation of a shock between the core and the envelope.

To account for the number of red supergiants in the LMC, the typical massive star must spend an appreciable time in the red before turning to the blue (Maeder 1987). If SK -69 202 followed this pattern, it must have evolved from the red to the blue. Depending on the details of the change of the core radius, an evolutionary calculation could end up on the hotter or the cooler side of the peak of one of the constant luminosity curves in Figure 2. Note also that two different evolutionary or structure codes could give very different solutions, depending on the exact input physics, and perhaps the numerical treatment. If the solution falls near one of the peaks in Figure 2, then one code could give a blue solution, and another could give a slightly smaller luminosity, or slightly larger core radius, and find only a solution on the Hyashi track. Similarly, variation of input physics in a single code, i. e. composition, could cause swings from the red to the blue as the critical points illustrated in Figure 2 are encountered. This may account in part for the presentation of some evolutionary tracks for SK -69 202 that stay in the blue (Arnett 1987a, Hillebrandt et al 1987) while others swing to the red and back to the blue (Woosley, Pinto and Ensman 1987, Wood and Faulkner 1987).

Consideration of the structure equations also gives an interesting constraint on the mass of the hydrogen envelope of the progenitor. Figure 4 gives the temperature profile as a function of mass for models with the envelope composition, $L=55,000$ Lo corresponding to a core mass of $\mathrm{MHe}_{\mathrm{He}}=4 \mathrm{M}_{\odot}, \mathrm{T}_{\text {eff }}=15,500 \mathrm{~K}\left(\mathrm{R}=33.4 \mathrm{R}_{\odot}\right)$, and a range of values of the total mass, $M$, from 7 to $18 \mathrm{M}_{\odot}$. Note that the temperature gradient is shallow for large masses but that it becomes ever steeper as the total mass is decreased. For a given core mass, $\mathrm{MHe}_{\mathrm{He}}$, this has the result that the temperature at that point, $\mathbf{T}\left(\mathrm{M}_{\mathrm{He}}\right)$, has a maximum as a function of the total mass. This is because for large $M$ the temperature gradient is small, and for sufficiently small $M$, the temperature has insufficient range to rise, despite the steep gradient, before $M_{\mathrm{He}}$ is encountered.

Many of the models demand that the temperature at some mass cut above the helium core be in excess of the ignition temperature of hydrogen, $T_{H}$, and hence, the structure is self-inconsistent. For a helium core mass of $4 \mathrm{M}_{\odot}$, models with total mass in the range 4.05 to $14 \mathrm{M} \odot$, are excluded. This conclusion does not depend sensitively on the specific value adopted for $T_{H}$, since the temperature profiles tend to rise so steeply in the excluded range. Similar conclusions follow for models with larger luminosities and 


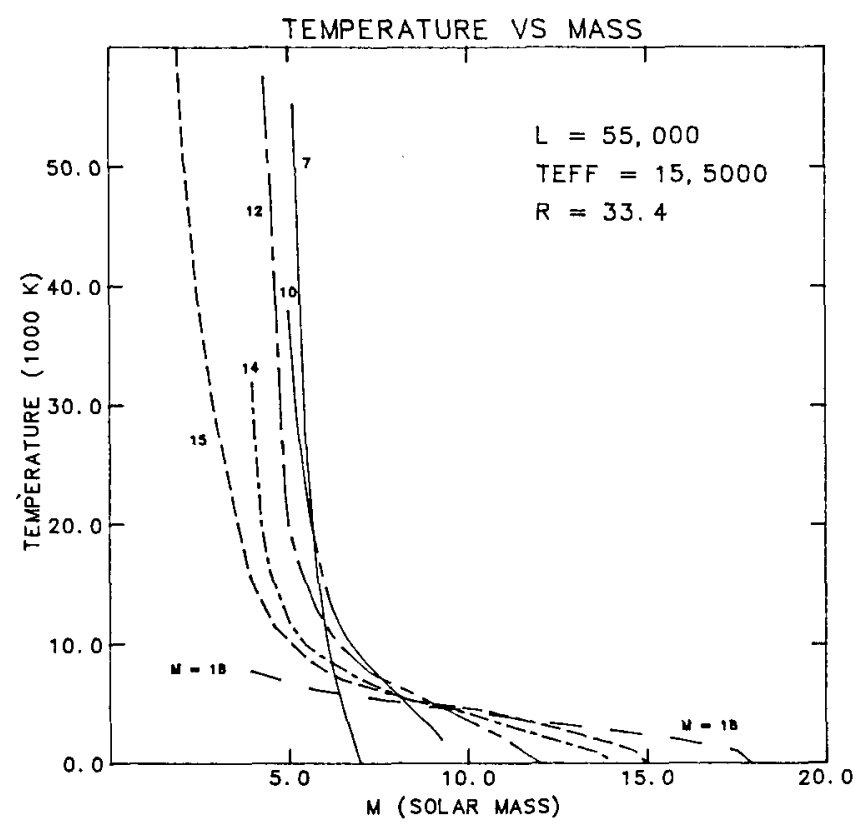

Figure 4. The temperature in units of $1000 \mathrm{~K}$ is given as a function of Lagrangian mass for models with $\mathrm{L}=$ $55,000 L_{\odot}, T e f f=15,500 \mathrm{~K}, \mathrm{R}=33.4 \mathrm{R}_{\odot}$ and envelope composition $X=0.7, Y=0.295, Z=0.005$, for a range of total masses. This luminosity corresponds to original main sequence mass of about $15 M_{\odot}$ and a helium core mass of $4 \mathrm{M}_{\odot}$. Note that for smaller total mass and hence smaller envelope mass the temperature rises rapidly to exceed the hydrogen ignition temperature well outside the core.

larger associated core masses. For $L=110,000 L_{\odot}$ and helium core mass of $6 \mathrm{M}_{\odot}$, the excluded range of mass is about 6.2 to $17 \mathrm{M}_{\odot}$.

We thus reach the conclusion that for the standard composition the mass of the envelope of SK -69202 must fall into two extreme ranges. Either the envelope mass is relatively large, corresponding to the star having reached the endpoint of its evolution with virtually all of its main sequence mass intact, or it is rather small, having suffered considerable mass loss. Intermediate values, which might be invoked by adopting arbitrary amounts of the ill-known mass loss, are excluded. This bifurcation in the allowed envelope masses for given core mass accounts for the extreme range of models which have been proposed. In Arnett (1987a) the envelope mass is about $11 \mathrm{M}_{\odot}$ for a core of $4 \mathrm{M}_{\odot}$, and in Wood and Faulkner (1987) the envelope mass is only about $0.2 \mathrm{M}_{\odot}$ for a core of $5.3 \mathrm{Mo}$. The present conclusions may depend to some extent on the assumption of strict thermal equilibrium which may fail in realistic evolution calculations. The range of excluded mass is smaller if the envelope is helium rich, and indeed any envelope mass is allowed by this criteria if $Y$ exceeds 0.5 (Barkat and Wheeler 1988). This point will be the subject of further investigation.

The proper structural solution for SK -69 202 can, in principle, be obtained from evolutionary calculations, or from observations of the light curve and spectra of the supernova itself. A basic constraint from the spectra is the observed Doppler shifts of the 
optical and infrared lines of hydrogen. The Balmer lines are observed to move as slowly as $5000 \mathrm{~km} / \mathrm{s}$ and the IR lines as slowly as $2000 \mathrm{~km} / \mathrm{s}$ (Menzies et al. 1987, Blanco et al. 1987, Phillips, private communication). We have computed dynamical models for stars of 15-20 Mo with their main sequence mass virtually intact and kinetic energy $\geq 1051$ erg/s which give minimum velocities for the hydrogen envelopes $\sim 2000 \mathrm{~km} / \mathrm{s}$, in accord with the observations. In contrast, models which reproduce the observed properties of SK -69 202 with small mass hydrogen envelopes produce velocities in the envelope far in excess of the observed minima. For induced kinetic energy of $6.6 \times 10^{50}$ ergs, the minimum velocity of the hydrogen in a model with a core of $6 \mathrm{M}_{\odot}$ and an envelope of 0.1 $M_{\odot}$ is $6510 \mathrm{~km} / \mathrm{s}$, on the high side of the observations. For a kinetic energy of $0.95 \mathrm{x}$ 1050 ergs the minimum hydrogen velocity is $2100 \mathrm{~km} / \mathrm{s}$, in keeping with the observations, but the early luminosity is very low. A model with a large hydrogen envelope to tamp the core expansion with a reverse shock gives a very slowly expanding core. This releases the radioactive heat slowly and can give a reasonable representation of the rise to maximum and decline to the radioactive tail (Woosley, Pinto, and Ensman 1987, Nomoto et al. 1987 ). For instance, we have calculated a model with a total mass of $15 \mathrm{M}$ and a kinetic energy of $1.4 \times 10^{51}$ ergs which gives a core energy of only $0.59 \times 10^{50}$ ergs. The light curves of both the low mass envelope models with 6 Mo cores just mentioned rise too quickly to maximum due to the rapid expansion of the core, despite the low induced energy. These models can thus be ruled out on the basis of the high hydrogen velocity, the rapid expansion, or both. The same is presumably true for the model presented by Wood and Faulkner (1987) with a core mass of $5.2 \mathrm{M}_{\odot}$ and envelope mass of $0.2 \mathrm{M}$.

\section{MODEL ATMOSPHERES}

SN 1987A also gives an important opportunity to explore the process of radiative transfer in supernovae. There are features in the spectrum that are special to SN 1987A, and others that are general to Type II supernovae. A careful study of the spectra of SN 1987A should provide a deeper understanding of that particular event, and of the principles to be applied to other supernovae. Excellent spectral coverage has been provided by observatories throughout the world. Optical spectra have been presented by Tyson and Boeshaar (1987), Danziger et al. (1987), Menzies et al., (1987), Blanco et al. (1987), Ashoka et al. (1987), Catchpole, et al. (1987) and Hanuschik and Dachs (1987). Ultraviolet spectra have been presented by Wamsteker et al. (1987), Cassatella, et al. (1987) and by Kirshner et al. (1987). There is also a growing body of infrared data, but that will not be considered in detail here.

The optical spectra for the first 4 days while the supernova was rising to an initial local maximum were rather simple, showing broad Balmer lines. After two days (circa February 25), the spectrum showed $\mathrm{He} I \lambda 5876$ but no sign of $\mathrm{Ca} \mathrm{H}$ and $\mathrm{K}$ or the IR triplet, due, presumably, to the high temperature that ionizes $\mathrm{Ca}$ Il. After four days (circa 
February 27) $\mathrm{Ca} \mathrm{H}$ and $\mathrm{K}$ were readily apparent, but the $\mathrm{He} I$ line had faded. After about 10 days (circa March 5) structure due to $\mathrm{Fe}$ II and $\mathrm{Na} \mathrm{D}$ appeared. A few days later a line appeared at $6070 \AA$ which Williams (1987) attributes to Ba II. Up to this time, the lines of the Balmer series became deeper, shifting to lower velocities, with $\mathrm{H}$ alpha showing a growing net emission. There was no discernible Balmer jump in absorption or emission (Dopita, Menzies, private communication). After about 10 days the Balmer lines began to fade rapidly. $\mathrm{H} \beta$ and $\mathrm{H} \gamma$ were barely discernible after 20 days. The optical spectrum did not change qualitatively for the next four months as the supernova passed through maximum in mid-May. $\mathrm{H} \beta$ did re-emerge with a P-Cygni profile around the end of April (65 days) (Catchpole et al. 1987).

The evolution of the spectrum of SN 1987A in the ultraviolet was extremely rapid and unique for an SN II. For the first day or so the spectrum was dominated by broad features from 1200 to $3200 \AA$ which were unlike any previous observed supernova (Wamsteker et al. 1987, Kirshner et al. 1987). By the third or fourth day, the spectrum resembled that of previously observed SN la and SN lb. Wheeler et al. (1986) attribute the UV spectra of SN I to resonant line scattering of Fe II, and hence a similar effect is presumably at work in SN 1987A. SN 1987A faded rapidly until it could no longer be seen at short wavelengths with the IUE satellite.

Various aspects of the formation of the optical and UV spectra of SN 1987A have been discussed by Fransson et al. (1987), Williams (1987), Branch (1987b), and Lucy (1987). Details of the current discussion will be given by Harkness and Wheeler (1988). Here we focus on the relatively simple spectrum when the supernova was two days old. As a simplifying assumption, we adopt a power law density structure $\left(\rho \propto r^{-n}\right)$ for the atmosphere. Branch (1987a) and Dopita et al. (1987) assume a constant opacity and hence that the optical depth and density both follow the same power law structure to relate the power law index to the rate of recession of the velocity of the matter at the photosphere. They derive $n \sim 11-15$ for the first four or five days and $n \sim 4-5$ during the subsequent few weeks. Models are calculated with $n=5,7$ and 11 . Homologous expansion is assumed and an inner velocity and hence radius is assigned corresponding to the selected epoch, eg, 2 days. The envelope composition is taken to be $X=.7438, Y=.2520$, and heavier elements $1 / 4$ of the solar distribution. The atmosphere models are calculated in the approximation of LTE. The calculation is first done in the locally co-moving frame in which the opacities can be solved exactly without concern for "expansion opacity" effects, and then transformed to the observer frame (Harkness 1985, 1986). All sources of continuum opacity are included and lines are treated by resonance scattering. Of order 200 lines are included in the calculations.

The velocities of the absorption minima of the Balmer lines are basically set by the density at a given velocity. The absorption minima are essentially independent of the luminosity of the model for reasonable values of the luminosity. At higher luminosity and temperature more hydrogen is ionized at lower radii and hence velocities, but more of the remaining hydrogen is excited, and these two effects are found to offset one another. Several aspects of the spectra do change significantly with the luminosity. One is the 
$\mathrm{H} \alpha$ line profile. The blue edge of the feature, which samples the highest velocity matter, is distinctly rounded in the observations. The brightest models reproduce this aspect rather well, whereas the dimmer models give sharper, abrupt blue edges. Other features sensitive to the luminosity are the $\mathrm{Ca} / \mathrm{l}$ lines, at $\mathrm{H}$ and $\mathrm{K}$ and the infrared triplet. These are not observed at two days, but appear in many of the theoretical spectra which are sufficiently dim or extended that appreciable Ca II exists. Satisfactory models must have the Ca II ionized away at this epoch.

No models with $n=5$ at two days are satisfactory. They are all plagued by very strong $\mathrm{Ca}$ Il lines and give colors that deviate considerably from those observed. The same is true to a lesser extent for the models with $n=7$. Of this series of models, the best agreement occurs for $n=11$. Figure 5 gives three models with $n=11$, a normalizing density of $1.5 \times 10^{-14} \mathrm{gm} / \mathrm{cm}^{3}$ for the matter moving at $20,000 \mathrm{~km} / \mathrm{s}$, at different luminosities. At two days, Menzies et al. (1987) give a bolometric luminosity for SN $1987 \mathrm{~A}$ of $4 \times 10^{41} \mathrm{erg} / \mathrm{s}$ for a distance modulus of 18.5 and. $A_{V}=0.6$. At $4 \times 10^{41}$ erg/s the $\mathrm{Ca}$ II lines are still rather strong in the models, whereas at $5 \times 10^{41} \mathrm{erg} / \mathrm{s}$, they have nearly disappeared. The higher luminosity is thus favored in this series, but a somewhat steeper density gradient would probably accommodate the lack of Ca II

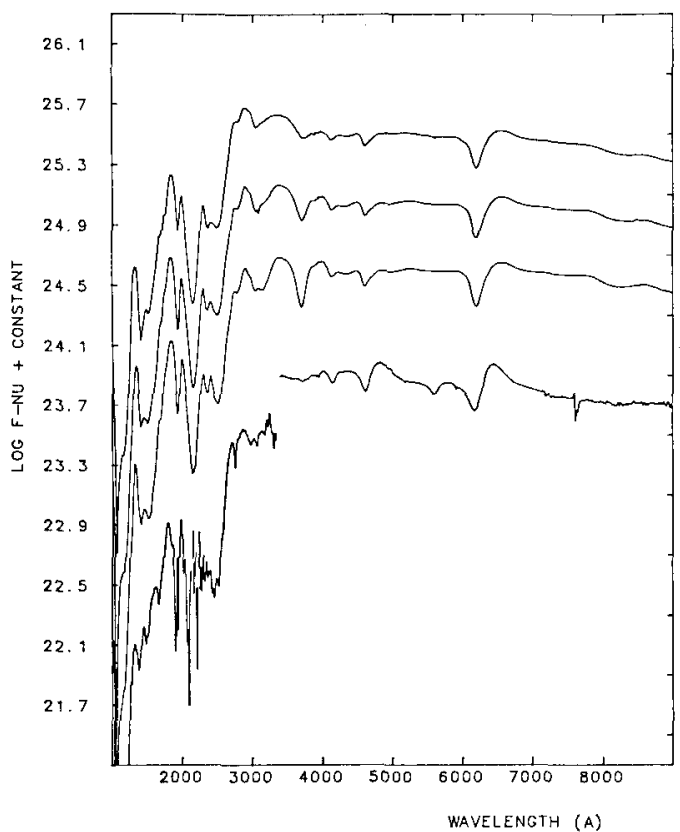

Figure 5. Three model atmospheres are given corresponding to a density profile of $r^{-11}$, with $\rho=1.5 \times 10^{-14}$ $\mathrm{gm} / \mathrm{cm}^{3}$ at $20,000 \mathrm{~km} / \mathrm{s}$ at two days. The three curves correspond to luminosities of $4.9,3.9$, and $3.2 \times 10^{41}$ $\mathrm{erg} / \mathrm{s}$, from top to bottom, respectively. The lower curves are optical spectra from Cerro Tololo provided by Mark Phillips and UV spectra provided by Bob Kirshner. Note the diminishing Ca II features with increasing luminosity, and the strong UV deficit which is unprecedented in a Type II supernova. 
features with a somewhat lower luminosity. At this epoch, the lack of the Ca II features thus serves as an intrinsic luminosity indicator.

As anticipated, the ultraviolet spectra are formed by resonant scattering of many Fe II lines. The resulting peaks are not emission lines, but rather the absence of blended $P$. Cygni absorption troughs in wavelength ranges where the density of $\mathrm{Fe} I \mathrm{Il}$ lines is less. This is particularly true of the peak at $1800 \AA$ which occurs at about $2000 \AA$ in the somewhat lower velocity environment of $S N$ la and $S N$ ib (Wheeler, et al. 1986). Mg II $\lambda 2797$ also makes a significant contribution to the minimum at $2400 \AA$. The success of these models in reproducing the UV spectrum suggests that "ordinary" Type II supernovae, which do not display such a UV deficit, have a more significant circumstellar nebula in the immediate environment of the supernova. This matter can dynamically affect the density gradient in the supernova ejecta, and produce a separate source of UV radiation.

\section{COMPARISON TO OTHER SUPERNOVAE}

The McDonald Observatory archives of supernova spectra have been examined to compare and contrast SN 1987A with other Type II supernovae. SN 1979C and SN $1980 \mathrm{~K}$ were two well-studied Type II events with "linear" light curves. There is controversy over whether linear SN II represent a distinct class or a part of a continuum for which well defined "plateau" events are another extreme. Near maximum light, SN $1979 \mathrm{C}$ and $1980 \mathrm{~K}$ showed nearly pure continua rather than the strong Balmer lines which characterized SN 1987A for the first several weeks. At about two months, SN $1980 \mathrm{~K}$, in particular, still displayed strong Balmer lines which had nearly disappeared from SN 1987A at a similar epoch. SN $1979 \mathrm{C}$ and 1980K show no sign of the "barium" line at $6070 \AA$. Whether these various differences mean that Type II linear events differ from SN 1987A in mass, composition, or excitation in the envelope is an important topic for future investigation.

SN 19861 in M 99 was the first supernova discovered by the Berkeley supernova search, and it has proved itself to be a classical plateau event. The spectra of SN 1987A do not at any epoch resemble the McDonald spectra of 19861 at about one month. There are some important similarities between the two events when they are about two months old, including some evidence in SN 19861 for the line at $6070 \AA$ in a spectrum obtained by $\mathrm{C}$. Foltz which is distinctly absent in the earlier McDonald spectra. Most of the similarities are due to ubiquitous features of $\mathrm{Fe} \mathrm{II}$, and hence they alone do not provide a solid link between SN 1986 I and SN 1987A.

The most intriguing spectrum in the McDonald archives in this regard is of $\mathrm{SN}$ $1985 \mathrm{H}$ in NGC 3359 obtained at an uncertain epoch. This spectrum is shown in Figure 6 along with a spectrum of SN 1987 A on April 28 kindly provided by Mike Dopita. There are strong similarities between these two spectra, most of which are again due to $\mathrm{Fe}$ II. The $H \beta$ feature is strong in this spectrum of $S N 1985 \mathrm{H}$. In SN 1987A it was just 


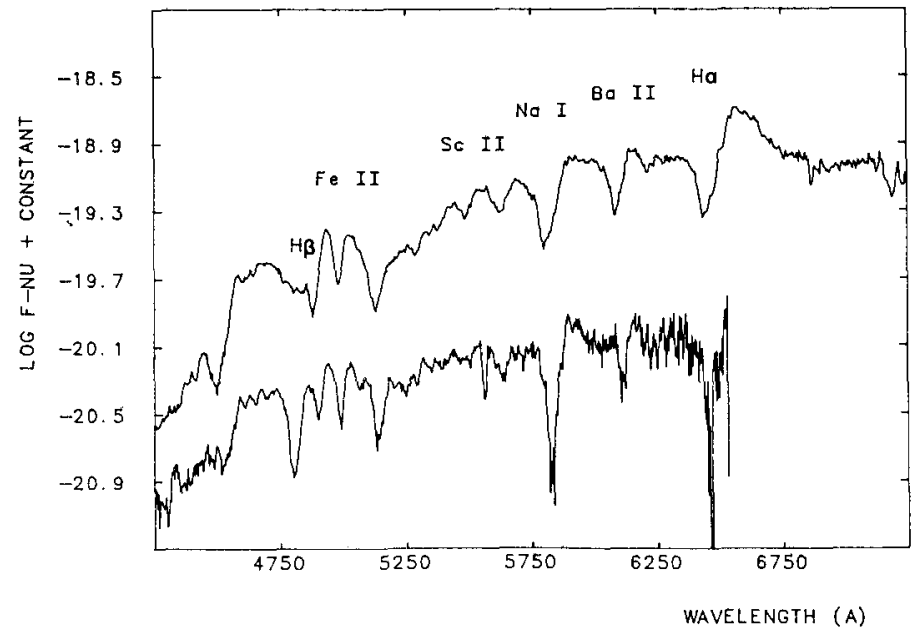

Figure 6. The spectrum of SN $1985 \mathrm{H}$ in NGC 3359 obtained by E. Barker and A. Cochran with the 2.7 meter telescope at McDonald Observatory on April 17, 1985 is shown in comparison with a spectrum of SN 1987A on April 28,1987 obtained at Siding Springs and provided by Mike Dopita. Note the similarity of the $\mathrm{H} \alpha$ absorption, $\mathrm{Na} \mathrm{D}$, the many $\mathrm{Fe} \| \mathrm{I}$ features in the blue and especially the strong absorption at $6070 \mathrm{~A}$.

beginning to strengthen at the epoch shown in Figure 6 and reached a strength comparable to that displayed here for $\mathrm{SN} 1985 \mathrm{H}$ about a month later, at the end of May. In general, the features in $\mathrm{SN} 1985 \mathrm{H}$ are narrower than those of the April 28 spectrum of SN 1987A, suggesting an epoch for the spectrum of SN $1985 \mathrm{H}$ of greater than two months. The most interesting feature is the strong line at $6070 \AA$, suggesting that $S N$ $1985 \mathrm{H}$ is closely related to SN $1987 \mathrm{~A}$.

At this writing, nothing is known about the photometric history of $\mathrm{SN} 1985 \mathrm{H}$, so it is not known how long after the explosion this spectrum was obtained, nor what luminosity should be expected. The supernova was at $V=16.4$ on April 12, 1985 (IAUC 4053). The distance modulus of NGC 3359 is about 30.21 according to de Vaucouleurs (private communication), so at the time the spectrum was obtained the absolute magnitude was about $M V=-13.8$ on this distance scale, neglecting reddening. The spectra of SN 1985H suggest that it is of order two months old (IAUC 4058), or older, as discussed above. Type II plateau events can remain on the plateau in $\mathrm{V}$ for about three months during which they decline 0.2 magnitudes (Doggett and Branch 1985). On the plateau they are about $\mathrm{MV} \sim-16$ to -17 on the short distance scale and 1.5 magnitudes brighter on the long scale. If $\mathrm{SN} 1985 \mathrm{H}$ is related to such events, it must have declined off the plateau by 2 to 3 magnitudes and hence be of order 150 days after maximum, independent of the distance scale. Over the temporal range of interest, $60-150$ days, SN $1987 \mathrm{~A}$ varied between $M_{V} \sim-16$ and -14.5 for $m-M=18.5$ and $A_{V}=0.6$. $S N 1985 \mathrm{H}$ would have been significantly dimmer than this on the short distance scale, but within this range on the long scale. Uncertainties in the epoch and distance of SN $1985 \mathrm{H}$ prevent any firm conclusion, but one can not on this basis rule out the notion that $\mathrm{SN}$ $1985 \mathrm{H}$ may have been of the same class of event as SN 1987A. 


\section{CONCLUSIONS}

Three different structures are found for stellar models with identical luminosity, total mass, core mass, and core radius as the effective temperature is varied. Two of these solutions can correspond to effective temperatures in the range pertinent to SK -69 202. This implies that modeis for the progenitor of SN 1987A will prove very sensitive to physical assumptions and numerical treatment in structural and evolutionary calculations.

Self-consistent models which match the observational constraints of SK -69 202 with envelopes of normal hydrogen and helium abundance suggest that the progenitor of SN 1987A must have undergone essentially no mass loss, or must have lost nearly all the hydrogen-rich envelope leaving $\sim 0.1 \mathrm{M}$. The models with smaller mass envelopes are shocked to excessive velocities in the core or envelope even with very low kinetic energy, and hence may be ruled out. The envelope composition may not be normal, but may be helium enriched. This avoids the problems of structural self-inconsistency, and may allow a range of envelope masses that are consistent with basic observational constraints.

The LTE models with $\rho$ or $r^{-11}$ give a reasonable representation of the optical and the "unique" UV spectra of SN 1987A at about 2 days. A lower limit to the luminosity is given by the Balmer line profiles and the absence of Ca II features. It is difficult to see how the current models could be reconciled with a distance modulus of 18.3 and/or an extinction significantly smaller than $A_{V}=0.6$, but this important issue deserves further study. Comparison of the light curve of SN 1987A to previous SN II plateau events suggests that either $\mathrm{H}_{0} \sim 100 \mathrm{~km} / \mathrm{s} / \mathrm{Mpc}$ or that $\mathrm{SN} 1987 \mathrm{~A}$ has ejected about 4 times less $56 \mathrm{Ni}$ than ordinary $\mathrm{SN}$ II plateau events.

At the close of this conference, we do not seem to have a completely self-consistent model in which the basic features of the light curve and spectra are satisfied by calculations of the theoretical spectra and light curves at all epochs. There is much left to be learned about the structure of the progenitor and the manner in which it exploded.

This research is supported in part by NSF Grant 8413301 , by the R. A. Welch Foundation, and by an allotment of computer time at the Center for High Performance Computing at the University Texas. We are grateful to Mike Dopita, Mark Phillips, and Bob Kirshner for providing us with optical and UV data in numerical form, and to Stan Woosley for some of his dynamical models. We have benefitted from conversations with numerous colleagues who have shared their excitement and insights into this remarkable event.

\section{REFERENCES}

Arnett, W. D. 1977, Ap. J. Suppl., 35, 145.

1987a, Ap. J., 319, 136.

1987b, preprint. 
Ashoka, B. N., Anupama, G. C., Prabhu, T. P., Giriidhar, S., Ghash, K. K., Jain, S. K., Pati, A. K., and Kameswara Rao, N. 1987, J. Astron. Astr., 8, 195.

Barkat, Z. and Wheeler, J. C. 1988, in preparation.

Blanco, V. M., et al. 1987, Ap. J., 320, 589.

Branch, D. 1987a, Ap. J. (Letters), 320, L23. 1987b, Ap. J. (Letters), 320, L121.

Cassatella, A., Fransson, C., van Santvoort, J., Gry, C., Talavera, A., Wamsteker, W. and Panagia, N. 1987, Astr. \& Ap., 177, L29.

Catchpole, R. M. et al. 1987, M.N.R.A.S., in press.

Chevalier, R. A. and Fransson, C. 1987, Nature, 328, 44.

Danziger, I. J., Fosbury, R.A.E., Alloin, D., Cristiani, S., Dachs, J., Gouiffes, C., Jarvis, B., and Sahu, K. C. 1987, Astr. \& Ap., 177, L13.

Doggett, J. B., and Branch, D. 1985, Ap. J., 90, 2303.

Dopita, M. A., Achilleos, N., Dawe, J. A., Flynn, C. and Meatheringham, S. J. 1987, preprint.

Fransson, C., Grewing, M., Cassatella, A., Panagia, N. and Wamsteker, W. 1987, Astr. \& Ap., 177, L33.

Grassbergh, E. K., Imshennik, V. S., Nadyozhin, D. K., and Utrobin, V. P. 1987, preprint.

Hanuschik, R. W. and Dachs, J. Astr. and Ap., 182, L29.

Harkness, R. P. 1985, in Supernovae as Distance Indicators, ed. N. Bartel (Berlin: Springer-Verlag), p. 183.

Harkness, R. P. 1986, in Radiation Hydrodynamics in Stars and Compact Objects, ed. D. Mihalas and K.-H.A. Winkler (Berlin: Springer-Verlag), p. 166.

Harkness, R. P. and Wheeler, J. C. 1988, in preparation.

Hillebrandt, W., Höflich, P., Truran, J. W., and Weiss, A. 1987, Nature, 327, 597.

Kirshner, R., Sonneborn, G., Crenshaw, D. M., and Nassiopoulos, G. E. 1987, Ap. J., $320,602$.

Lucy, L. B. 1987, Astr. \& Ap., 182, L31.

Maeder, A. 1987, Proceedings of the ESO Conference on SN 1987A, in press.

Menzies, J. W., et al. 1987, M.N.R.A.S., 227, 39p

Nomoto, K., Shigeyama, T., and Hashimoto, M. 1987, in Proceedings of the ESO Workshop on SN 1987A, in press.

Shigeyama, T., Nomoto, K., Hashimoto, M., and Sugimoto, D. 1987, Nature, 328, 320.

Tyson, J. A. and Boeshaar, P. C. 1987, preprint.

Walborn, N. R., Lasker, B. M., Laidler, V. G., and Chu, Y.-H. 1987, Ap. J. (Letters), 321, L41.

Wamsteker, et al. 1987, Astr. \& Ap., 177, L21.

Wheeler, J. C., Harkness, R. P., Barkat, Z. and Swartz 1986, P.A.S.P., 98, 1018.

Williams, R. E. 1987, Ap. J. (Letters), 320, L117.

Wood, P. R. and Faulkner, D. J. 1987, preprint.

Woosley, S. E., Pinto, P. A., Martin, P. J., and Weaver, T. A. 1987, Ap. J., 318, 664.

Woosley, S. E., Pinto, P. A., and Ensman, L. 1987, Ap. J., in press. 